\title{
Rotation mentale et ordinateur
}

\author{
MÁRIA BAKÓ
}

Résumé. P. H. Maier, en se basant sur des recherches psychologiques, distingue cinq éléments d'intelligence spatiale. Dans cet article nous étudions comment l'ordinateur peut intervenir dans le développement de l'élément rotation mentale. Nous avons écrit des logiciels qui utilisent les rétroactions perspectives et nous avons effectué des expériences avec cinq groupes d'éleves. Pour pouvoir poursuivre le travail et le développement de travail de chaque éleve et pour faciliter l'examen des résultats, les tests sont au format HTML et les réponses des éleves ont été traitées par un serveur central. La statistique montre que les résultats des éleves s'améliorent au fur et a mesure.

Key words and phrases:

ZDM Subject Classification:

\section{Introduction}

Depuis plus de cinquante ans, les mathématiciens et les philosophes, s'intéressent particulierement aux questions de vision spatiale, d'orientation spatiale et a la notion de l'image mentale. Selon Cooper et Shepard l'image mentale est un substitut de la maquette, "les images mentales représentent la structure tridimensionnelle des objets et non les caractéristiques bidimensionnelles des dessins » [3]. Kosslyn s'est intéressé a l'exploration mentale de plans et de cartes, et il a montré que la durée de cette exploration était proportionnelle aux distances réelles sur la carte [6]. Shepard a travaillé sur des dessins d'assemblages de cubes, dont il fallait déterminer s'ils étaient ou non identiques, a une rotation pres. Il montre que « la détermination de l'identité des formes est comme une sorte de rotation

Copyright (C) 2004 by University of Debrecen 
mentale dans l'espace a trois dimensions qui s'effectue avec un vitesse d'environ $60^{\circ}$ par second » [12]. Hartmann et Reiss examinent les stratégies de résolution utilisées par les éleves dans le cas de la géométrie dans l'espace [5, 11].

En 1992 Howard Gardner a édifié la théorie de l'intelligence multiple, selon laquelle il existe huit intelligences qui peuvent etre considérées comme des facultés créatrices et des facultés de résolution. L'intelligence spatiale, que l'on utilise pour visualiser et dessiner, est une des composantes de cette intelligence multiple.

D'apres H. Gardner l'intelligence spatiale peut etre un avantage inestimable dans notre société compétitive. La faculté spatiale est tres complexe; c'est pourquoi Peter Herbert Maier, en se basant sur des recherches psychologiques, distingue cinq éléments d'intelligence spatiale [7] :

- La perception spatiale, qui demande de distinguer les directions horizontale et verticale malgré les informations perturbatrices.

- La visualisation, qui comporte la capacité de représenter une configuration dans laquelle les éléments composants bougent ou se déplacent.

- La rotation mentale, qui comporte la capacité de faire tourner (en mémoire) vite et correctement les figures bi- et tridimensionnelles.

- Les relations spatiales, qui comportent la capacité de comprendre la configuration spatiale des objets ou parties d'un objet et ses relations entre eux.

- L'orientation spatiale, capacité de s'orienter physiquement et mentalement dans l'espace.

Selon P. H. Maier, dans l'enseignement de la géométrie dans l'espace on devrait développer ces éléments et les maquettes tridimensionnelles devraient se situer au centre de l'enseignement de la géométrie [8]. Mais la réalisation systématique d'une maquette est une opération couteuse (en moyens et en temps), c'est pourquoi souvent elle est négligée.

Une enquete ${ }^{1}$ du ministere de l'Education Nationale Française, menée a la fin de l'année scolaire 1985-86 au niveau de la classe de Seconde (âge 15 ans) montre qu'en mathématiques, la Géométrie de l'espace est, avec les Statistiques, l'une des deux parties «mal aimées ». Elle fait aussi apparaître que $10 \%$ seulement des professeurs avaient a ce moment abordé la géométrie dans l'espace. Si l'on essaie de déterminer les raisons de cette désaffection en interrogeant les enseignants de Seconde, l'argument le plus fréquemment évoqué est le manque de temps. En

${ }^{1}$ cité par Parzysz [10, p. 5] 
réalité, la raison profonde est inavouable : la géométrie de l'espace passe pour etre un sujet difficile; parce que, dit-on, « les éleves ne voient pas dans l'espace».

Ce probleme est également tres bien présenté dans l'expérience effectué par un groupe de professeurs de l'IREM de Strasbourg [?].

Nous aimerions analyser comment l'ordinateur et les logiciels peuvent etre utiles dans le développement de cette «vision spatiale », plus précisément dans le développement de quelques éléments d'intelligence spatiale. Dans cet article nous étudions la question suivante : L'ordinateur peut-il aider a développer la rotation mentale?

Pour analyser cette question nous avons écrit des logiciels concernant les rotations spatiales d'un cube. Pendant les expériences les éleves ont eu la possibilité de faire des aller-retour entre la page WEB du test (ou ils ont donné peut-etre une réponse erronée) et la page contenant la réponse correcte. L'hypothese de notre recherche : une attitude réflexive de l'apprenant sur ses erreurs est-elle susceptible de lui permettre de les surmonter?

Dans le paragraphe suivant nous présentons le logiciel utilisé. Les paragraphes 3 et 4 comportent les expériences effectuées et les résultats de ces expériences. Dans les paragraphes 5 et 6 sont présentées les différences entre l'environnement du logiciel et l'environnement papier-crayon d'une part, la manipulation d'une maquette d'autre part. Le paragraphe 7 contient les résultats d'un questionnaire concernant l'avis des éleves sur le test. Le paragraphe 8 comporte les conclusions.

\section{Le logiciel}

En se basant sur les exercices qui ont été déja proposés par différents chercheurs en environnement papier-crayon, nous avons écrit des logiciels qui utilisent les rétroactions perspectives [2, p. 260].

Nous aurions aimé poursuivre le travail et le développement de travail du chaque éleve. Pour faciliter l'examen des résultats, les tests sont au format HTML et les réponses des éleves ont été traitées par un serveur central.

Les exercices ont été regroupés en cinq catégories. Le niveau de difficulté des exercices s'augmente. Nous aurions aimé que les éleves s'habituent a l'utilisation du logiciel et qu'ils n'aient pas d'erreurs causées par la méconnaissance du logiciel dans les niveaux plus difficiles, c'est pourquoi les deux premieres catégories ont été des exercices «d'échauffement ». Parmi les solides, nous avons choisi le cube car il est le solide le plus simple et plus connu. 
Nous avons choisi les catégories suivantes :

(1) symétrie par rapport un plan passant par le centre du cube parallelement a une face (selon la position du plan, 3 types de symétrie),

(2) symétrie par rapport un plan passant par deux diagonales de faces paralleles, (selon la position du plan, 6 types de symétrie),

(3) rotation d'ordre quatre (rotation autour de l'axe central du cube), (12 types de rotation : 3 positions d'axe, 2 sens et 2 angles $\left(90^{\circ}, 180^{\circ}\right)$ ),

(4) rotation d'ordre deux (rotation autour d'une droite passant par les milieux de deux aretes opposées) (12 types de rotation : 6 positions d'axe, 2 sens et 1 angle $\left(180^{\circ}\right)$ ),

(5) rotation d'ordre trois (rotation autour de la diagonale du cube) (8 types de rotation : 4 positions d'axe, 2 sens et 1 angle $\left(120^{\circ}\right)$ ).

Les rotations ou les sens sont différents mais ou les résultats sont identiques ont été considérées comme des problemes différents. En géométrie, une rotation de $180^{\circ}$, dans le sens positif ou dans le sens négatif, est considérée comme une seule et meme transformation. Ce n'est pas l'opinion qui est prise ici, puisqu'on prend en compte le mouvement de rotation, qui est différent dans les deux cas.

Tous les problemes comportent trois dessins et un texte qui précise la transformation. Le premier dessin représente un cube ABCDEFGH dont les lettres sont mises par l'ordinateur dans des ordres différents. Le deuxieme dessin est le dessin de la transformation en précisant le plan de la symétrie ou l'axe de la rotation. Le troisieme dessin représente un cube ou, a côté d'un sommet, nous avons mis un point d'interrogation. En ce sommet les éleves devaient taper le nom du sommet qui, apres la transformation, se trouvera dans cette position.

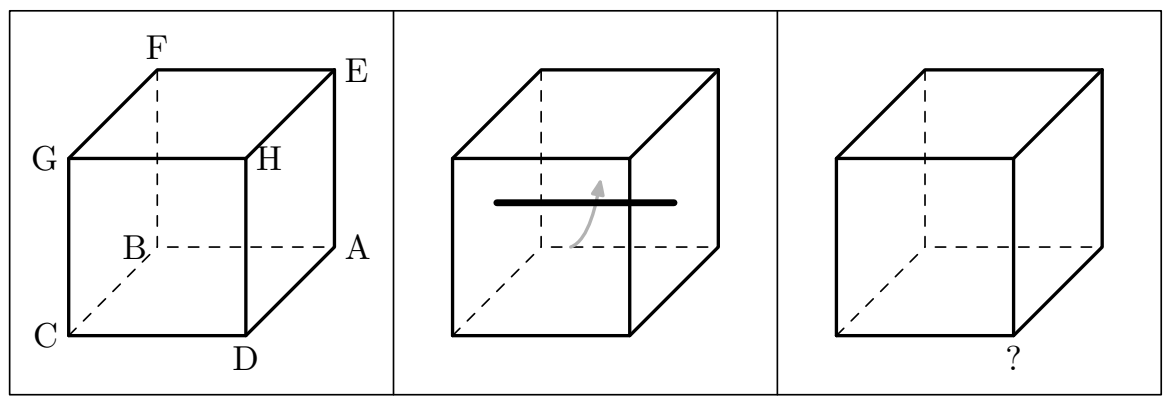

FIG. 1. DESSIN? 
Chaque page HTML contient sept problemes présentés sous cette forme. Si l'éleve a répondu a chaque probleme, alors en appuyant sur le bouton « soumettre »il peut envoyer les réponses.

Si l'éleve n'a laissé sans réponse aucune question et si toutes ses réponses ont été valables (il a choisi une lettre $\mathrm{A}-\mathrm{H}$ ) le logiciel (écrit en langage PERL) sur le serveur, selon le nombre de réponses correctes, donne les appréciations suivantes :

- sept réponses correctes : il félicite l'éleve et lui propose le niveau suivant,

- de quatre a six réponses correctes : le logiciel dessine les bonnes réponses (le premier cube, la transformation et le cube avec la réponse) dans les cas ou l'éleve s'est trompé; il lui propose de répéter le meme niveau avec des problemes différents,

- de zéro a trois réponses correctes : comme dans le cas précédent le program donne les réponses, puis il envoie l'éleve sur la page d'information, pour voir le cube en train de tourner, et il lui propose de répéter le niveau.

Si l'éleve donne des réponses non valables ou s'il laisse un probleme sans réponse, le logiciel lui demandera de corriger cette erreur et de donner une réponse valable.

Dans chaque cas, quand l'éleve a fait une erreur le logiciel les trois dessins du cube car nous souhaitions qu'il puisse regarder ensemble la position de départ du cube, la transformation et la position finale du cube pour mieux comprendre le probleme. Apres le test quelques éleves (3 sur 54) ont remarqué qu'ils avaient eu des difficultés avec l'interprétation de la position de la fleche montrant la direction de la rotation. Ils n'ont pas arrivés a discerner si la fleche passait au-dessus ou au-dessous de l'axe de rotation.

Comme l'écran de l'ordinateur est bidimensionnel on peut retrouver ici l'ambiguité de la représentation plane de l'espace [9]. Pour la géométrie dans l'espace en effectuant le dessin on «perd une dimension », c'est pourquoi le dessin est beaucoup plus éloigné, de la situation présentée dans l'énoncé d'un probleme, qu'en géométrie plane.

A partir du niveau 3 (problemes de rotation), nous avons présenté les rotations avant chaque niveau, sur une page HTML. En utilisant les possibilités offertes par le WEB, les cubes ont « réellement »tourné a l'écran. Dans le cas des rotations d'ordre quatre, bien connu, nous nous sommes contentés seulement de la présentation des cubes tournants, mais dans le cas des rotations moins connues, d'ordre deux et trois, nous avons marqué un sommet pour mieux visualiser la trajectoire du point. 
Comme nous ne savions pas d'avance combien de tours allait faire un éleve avant de réussir a passer au niveau supérieur, les exercices ont été engendrés aléatoirement.

Nous avons pris 24 positions de départ, 6 symétries, 12 rotations d'ordre 4, 12 rotations d'ordre 2, 8 rotations d'ordre 3. C'est pourquoi, pour un meme niveau, nous pouvons avoir de 72 a 288 problemes différents. Grâce a ces grands nombres, les exercices se sont rarement répétés et les éleves ne pouvaient pas copier les réponses du voisin. La probabilité pour qu'un éleve trouve les bonnes réponses en tapant une lettre au hasard (entre A et $\mathrm{H}$ ), est pour chaque niveau. C'est pourquoi nous pensons qu'ils devaient réellement résoudre les problemes.

Le logiciel JavaScript qui a engendré les exercices et les procédés de vérification des réponses a été intégré dans la source de la page HTML. A l'aide de ces logiciels, le logiciel en PERL, sur le serveur, a permis de représenter les transformations qui ont été mal résolues et de nous envoyer les données en e-mail pour faire les statistiques.

Comme le logiciel est un élément important du milieu a-didactique [1], il est important de prendre en compte les contraintes spécifiques a l'environnement informatique. Une contrainte importante est la bidimensionalité de l'écran. L'autre contrainte importante est que le logiciel, dans le cas de la symétrie, ne permet pas d'obtenir des images mobiles.

\section{L'expérience}

L'expérience a eu lieu au lycée Fazekas Mihály de Debrecen en octobre 2002. Selon les possibilités d'acces aux salles d'informatique, avec l'aide de ma collegue Kiszely Ildikó, nous avons formé quatre groupes. L'expérience a été répétée le 10 mars 2003 avec l'aide de $M^{\text {me }}$ Janine Amiot au Lycée Victor Hugo de Colomiers (pres de Toulouse).

I. douze éleves a l'age de dix-huit ans dans une classe de spécialité informatique

II. quatorze éleves a l'age de treize ans dans une classe de spécialité mathématique

III. dix-huit éleves a l'age de quinze ans dans une classe de spécialité mathématique

IV. dix éleves a l'age de dix-sept ans dans une classe de spécialité mathématique 
V. quinze éleves (des éleves français) a l'age de 17 ans dans une classe d'orientation art-plastique

Les éleves, grâce au cours d'informatique, sont déja habitués a l'utilisation des ordinateurs. Comme l'utilisation des logiciels est tres simple (ils doivent uniquement taper sur un bouton du clavier et avec la souris cliquer sur le bouton « soumettre »), les éleves ont appris a l'utiliser en cinq minutes. Comme les transformations du plan et de l'espace étaient connues des éleves, ils avaient les connaissances nécessaires pour résoudre les problemes. Ils ont eu quarante minutes pour résoudre les cinq niveaux du test et pour répondre au petit questionnaire situé a la fin du test et accessible a partir de la premiere page. Le test original se trouve a l'adresse WEB : http://www.inf.unideb.hu/ aszalos/kocka.

\section{Les résultats}

Dans le Tableau 1 nous avons noté pour chaque groupe les niveaux et le nombre d'éleves qui ont réussi a résoudre ce niveau (mais pas le niveau suivant). Par exemple, sous la rubrique niveau $2 / \mathrm{III}$ on trouve 1 , qui signifie que dans le groupe III. il y avait un seul éleve qui a réussi a résoudre les problemes de réflexion (niveau 1 et 2) mais il n'a pas réussi ou n'a pas eu envie de résoudre les problemes de rotation (niveau 3). Dans la deuxieme colonne on n'a pas indiqué l'éleve n ${ }^{\circ} 24$ car il n'a meme pas réussi le premier niveau. Le Tableau 1. nous montre aussi que les groupes I. et IV. ont été les plus efficaces, car dans ces groupes chaque éleve est arrivé au moins au niveau 3. La répartition des éleves dans les groupes II. et III. est plus uniforme. Dans ces groupes il y a des éleves qui ne sont pas arrivés sur les niveaux des rotations (niveaux 3, 4 et 5). Dans le cas du groupe V. le nombre des éleves qui ont réussi le niveau 3 est tres élevé. Il est probable que s'ils avaient eu plus de temps ils auraient pu faire le (ou les) niveau(x) supérieur(s).

Tableau 1. Répartition des éleves selon le niveau atteint

\begin{tabular}{|l|r|r|r|r|r|}
\hline & I & II & III & IV & V \\
\hline niveau 1. & 0 & 2 & 3 & 0 & 2 \\
\hline niveau 2. & 0 & 4 & 1 & 0 & 2 \\
\hline niveau 3. & 2 & 3 & 6 & 1 & 9 \\
\hline niveau 4. & 3 & 2 & 4 & 2 & 2 \\
\hline niveau 5. & 7 & 2 & 4 & 7 & 0 \\
\hline Total & 12 & 13 & 18 & 10 & 15 \\
\hline
\end{tabular}


Le temps n'était pas mesuré a partir du début du cours, ni a partir de l'ouverture de la page HTML mais a partir de la réception de la premiere réponse. C'est la raison pour laquelle, si nous regardons les données, nous pouvons observer que les éleves d'un groupe commençaient le test a différents moments. Les éleves qui avaient passé plus de temps a lire les textes ou a réfléchir ont commencé plus tard, car ils ont donné les résultats du premier niveau plus tard que les autres.

Comme nous l'avons déja mentionné, les deux premiers niveaux ont été choisis de telle sorte que les éleves puissent s'habituer a l'utilisation du logiciel. Néanmoins, beaucoup d'éleves ont rencontré des difficultés dans ce domaine.

Dans cette expérience, nous n'avons pas aidé les éleves qui ont eu du mal a résoudre un probleme car nous voulions savoir jusqu'ou ils arriveraient avec la seule aide de l'ordinateur. Comme les résultats montrent, on ne peut pas les confier totalement aux ordinateurs car s'ils ont des difficultés, apres un certain temps ils abandonnent. Leur nombre diminue avec l'age, mais les expériences concernant l'enseignement a distance [4] montrent qu'il est tres difficile d'apprendre uniquement a l'aide de l'ordinateur.

Nous avons constaté que l'efficacité des éleves qui utilisent seulement l'ordinateur, sans explications du professeur, s'améliore avec le temps. Plus précisément le nombre d'erreurs sur un niveau diminue avec le temps. Par exemple dans le cas de l'éleve $\mathrm{n}^{\circ} 26$ cette diminution s'est réalisée a tous les niveaux (niveau 1:6, 0 ; niveau $2: 0$; niveau $3: 5,5,2,0$; niveau $4: 1,0$ ). Cependant si nous regardons les résultats de l'éleve $\mathrm{n}^{\circ} 21$ au premier niveau, nous pouvons remarquer qu'il a commis $7,4,4,6,3,3,3,6,6,7,1,2,0$ erreurs consécutives. Le nombre des réponses erronées est assez varié, il diminue, il augmente, il diminue etc. Pour pouvoir totaliser ces réponses de différents types nous procédons de la maniere suivante : pour chaque éleve, a chaque niveau, on prend la différence des nombres d'erreurs consécutifs. On prendra le nieme nombre en effectuant la soustraction des nombres d'erreurs commis entre le $(n+1)$ ieme et nieme essais. Si nous regardons les résultats de l'éleve $\mathrm{n}^{\circ} 26$ nous pouvons constater qu'il est tres proche du cas idéal ou les nombres d'erreurs diminue avec les essais; alors toutes les différences successives sont négatives (niveau $1:-6$; niveau $3: 0,-3,-2$, niveau $4:-1$ ). Dans le cas de l'éleve $n^{\circ} 21$ ces nombres seront : $-3,0,2,-3,0,0,3$, $0,1,-6,1,-2$. Pour chaque groupe, pour chaque niveau, pour chaque pas, on a additionné ces nombres. Sous les rubriques, le premier nombre $\left(a^{k, n}\right)$ indique la somme de ces nombres, le nombre entre parentheses le nombre de nombres additionnés. $a^{k, n}=\sum_{i}\left(e_{i}^{k, n+1}-e_{i}^{k, n}\right)$ ou $e_{i}^{k, n}$ sont les nombres des erreurs de l'éleve $i$ sur le niveau $k$ apres le $n^{\text {ieme }}$ essai. 
Tableau 2. La variation des erreurs (Groupe III.)

\begin{tabular}{|l|r|r|r|r|r|r|}
\hline Niveau/n & 1 & 2 & 3 & 4 & 5 & 6 \\
\hline niveau 1. & $-8(8)$ & $5(6)$ & $-10(4)$ & $-7(2)$ & $-1(1)$ & \\
\hline niveau 2. & $-2(8)$ & $-7(5)$ & $-1(2)$ & & & \\
\hline niveau 3. & $-3(5)$ & $-7(3)$ & $-1(1)$ & & & \\
\hline niveau 4. & $-17(9)$ & $4(4)$ & $-6(3)$ & & & \\
\hline niveau 5. & $-9(4)$ & $2(3)$ & $-1(1)$ & $2(1)$ & $-2(1)$ & $-1(1)$ \\
\hline
\end{tabular}

On doit mentionner ici que d'autres éléments comme la fatigue, les exercices fournis par le logiciel etc. peuvent aussi avoir une influence sur ces résultats.

En regardant par exemple les résultats du Groupe III. (Tableau 2.), nous pouvons remarquer que, dans la plupart des cas, le nombre retenu est négatif, ce qui signifie que petit a petit l'efficacité moyenne des éleves s'accroît.

Dans les Groupes I, II, IV et V les résultats sont semblables aux résultats du groupe III.

L'étape suivante se propose d'analyser le taux de réussite de chaque groupe pour chaque niveau.

A partir du troisieme niveau, il y a des exercices concernant les rotations. Nous ne pouvons pas dire que sur le troisieme niveau les exercices ont été tres difficiles, car beaucoup d'éleves les ont résolus sans erreur. Tous les éleves (59) qui avait terminé le niveau précédent, ont essayé de résoudre les exercices de ce niveau. Néanmoins, comme le nombre d'éleves diminue, les erreurs provenant de l'échantillonnage statistique augmentent. Sur le Dessin 2 on peut voir les douze rotations proposées a ce niveau.

A l'examen du Dessin 2 et du Tableau 3 nous pouvons conclure qu'il y a plus d'erreurs dans le cas des rotations de $180^{\circ}$ (les rotations $2,3,6,7,10,11$ ) que dans le cas des rotations de $90^{\circ}$. Ces dernieres sont probablement considérées comme plus faciles car tous les sommets du cube ne se déplacent que d'une position. Comme dans les deux niveaux antérieurs, les éleves du Groupe II (septieme) ont commis plus d'erreurs que les autres. Les autres groupes ont eu de meilleurs résultats dans les cas ou l'axe de rotation était vertical. Il est aussi intéressant de remarquer que les éleves hongrois ont eu davantage de problemes avec les rotations de $180^{\circ}$, alors que les éleves français ont commis plus d'erreurs dans les cas 1,4 et 8 , quand l'angle de rotation était de $90^{\circ}$ et l'axe horizontal. Tableau 3 Proportion d'erreurs au troisieme niveau du test

La statistique montre que les résultats des éleves s'améliorent au fur et a mesure. Les éleves qui ont eu 5 ou 6 erreurs au premier essai sont arrivés au 
Tableau 3. DESSIN 1

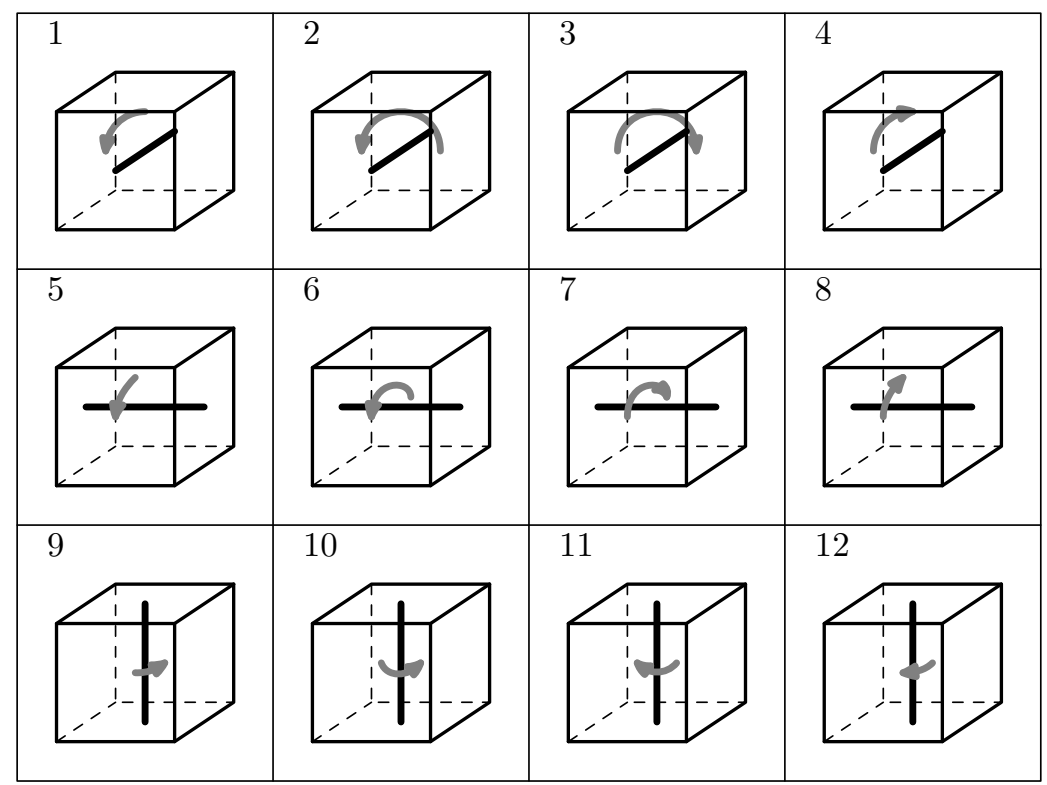

niveau apres quelques tentatives. Pendant l'expérience on a observé les éleves. S'ils ont commis des erreurs, ils ont fait plusieurs aller-retour entre la page WEB du test (avec la réponse erronée) et la page contenant la réponse correcte. Ils ont essayé de déchiffrer la cause de l'erreur en faisant des manipulations avec la main (simulant un cube imaginaire). En faisant les aller-retour et les manipulations, les éleves ont en général compris la cause de leur erreur et ils ont réussi a résoudre les exercices. Quelquefois on les a entendus dire « Ah, j'ai compris! Ça marche comme ça ». Ceci tend donc a confirmer l'hypothese de recherche signalée plus haut, et d'en attribuer possiblement la cause a l' « amélioration »de l'image mentale de la rotation du cube chez les éleves.

\section{L'environnement du logiciel et l'environnement papier-crayon}

L'analyse des manuels et livres d'exercices nous a montré que le nombre d'exercices pour chaque type de probleme est limité et qu'il y a tres peu de problemes concernant la vision spatiale. L'éleve qui a résolu un devoir a la maison ou un exercice de contrôle aura la réponse le lendemain ou la semaine d'apres. Le 


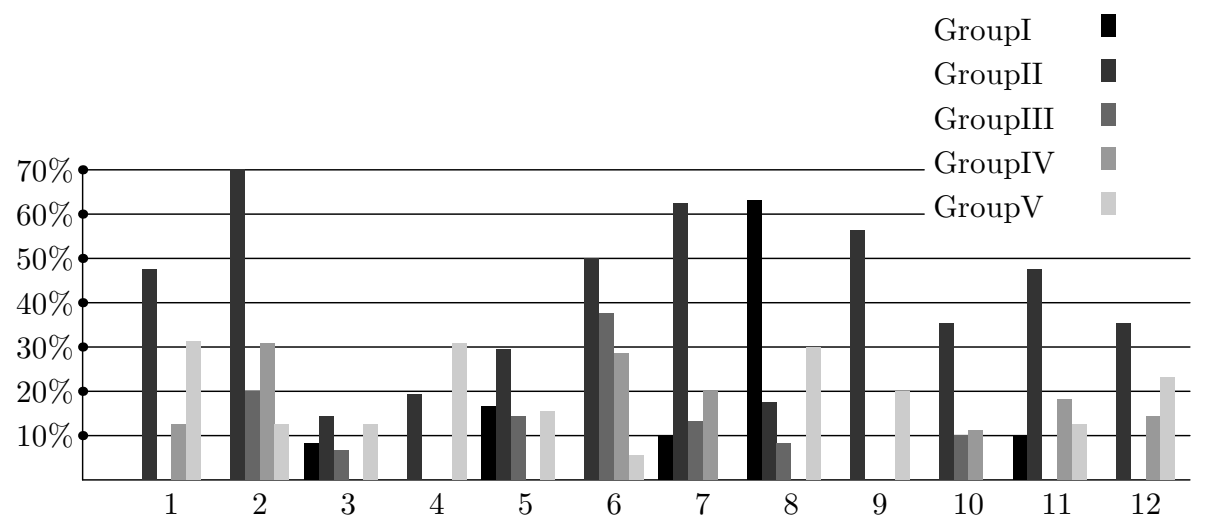

DEssin 2. !!!!

logiciel, lui, peut engendrer plus de 2000 exercices pour chaque type de probleme, en réduisant les répétitions éventuelles au minimum; de plus, il donne les réponses immédiatement. L'éleve ne peut passer au niveau $(n+1)$ que s'il a résolu sans erreur les sept exercices du niveau $(n)$. Comme on l'a déja mentionné, la probabilité pour que les éleves trouvent les bonnes réponses au hasard est tres petite, pour chaque niveau. Dans un environnement papier-crayon le professeur ne peut pas suivre continuellement les progres de chaque éleve pour chaque type de probleme. Le professeur peut observer (de loin) et peut aider personnellement chaque éleve en difficulté pendant l'utilisation du logiciel. Les éleves qui n'ont pas de difficultés peuvent avancer seuls et ils ne doivent pas subir les explications répétées du professeur. Les éleves peuvent se rendre compte de leurs progres, d'autre part, le professeur, en dressant les bilans, peut suivre les progres et les lacunes de ses éleves.

L'avantage du logiciel sur l'environnement papier-crayon réside également dans les possibilités du logiciel qui peut montrer le cube en mouvement. Dans les cas les plus difficiles, un sommet a été marqué pour pouvoir mieux suivre sa trajectoire.

\section{L'environnement du logiciel et la manipulation d'une maquette}

En utilisant une maquette, la réponse sera lisible immédiatement apres la manipulation, mais on n'aura qu'un nombre limité d'exercices. Un autre probleme 
pourrait etre la mauvaise interprétation d'une transformation; dans ce cas-la, l'éleve risque d'accepter une réponse erronée comme réponse correcte.

Pour les niveaux 1 et 2, nous avons proposé des symétries par rapport a un plan passant par le centre du cube parallelement a une face et des symétries par rapport a un plan passant par deux diagonales des faces paralleles. Sur une maquette, la symétrie par rapport a ces plans peut causer des difficultés pratiques.

\section{Le questionnaire}

A la fin du test il y avait un petit questionnaire contenant trois questions et une rubrique remarques, dans laquelle nous avons demandé aux éleves de noter toutes leurs remarques concernant le test. Parmi les 69 éleves, seuls 43 ont répondu.

Les questions ont été de type QCM. Les possibilités de réponses a la premiere question : Est-ce que tu aimerais résoudre de nouveau ce test? ont été les suivantes :

1. «J'aimerais les voir comme le milieu de mon dos. »(= Non, pas du tout.)

2. Pas dans le futur proche.

3. Oui, s'il y a de nouveaux tests.

4. Oui, maintenant si c'est possible.

Les opinions sont tres différentes. Un tiers des éleves (12 sur 43) disent qu'ils ne les ont pas aimés et ils ne veulent pas les retrouver. Néanmoins plus de la moitié (22 sur 43) d'entre eux ont apprécié le test et selon eux on pourrait poursuivre cette forme d'enseignement.

Tableau 4. !!!

\begin{tabular}{|l|r|r|r|r|}
\hline Réponse & 1 & 2 & 3 & 4 \\
\hline Nombre de réponses & 12 & 9 & 18 & 4 \\
\hline
\end{tabular}

Nous avons calculé la moyenne des nombres correspondant aux réponses pour chaque groupe et pour chaque niveau. Par exemple, dans le Groupe I, deux éleves (le nombre en parentheses) qui ont terminé le niveau 4 ont répondu aux questions. L'un a donné la réponse $n^{\circ} 3$, l'autre la réponse $n^{\circ} 2$. la moyenne est $(2+3) / 2=2,5$. En raison du petit nombre de réponses, les résultats sont assez dispersés; on peut néanmoins observer la tendance selon laquelle les éleves qui ont réussi le mieux sont plus favorables a la répétition du test. 
Tableau 5. !!!!

\begin{tabular}{|l|r|r|r|r|r|}
\hline Groupe/niveau atteint & Niveau 1 & Niveau 2 & Niveau 3 & Niveau 4 & Niveau 5 \\
\hline Groupe I & & & $2(1)$ & $2,5(2)$ & $2,6(7)$ \\
\hline Groupe II & & $2,3(3)$ & $2,5(2)$ & $3(1)$ & $3,5(2)$ \\
\hline Groupe III & $1,5(2)$ & $1(1)$ & $1,3(3)$ & $1,5(2)$ & $3(2)$ \\
\hline Groupe IV & & & & & $2,5(6)$ \\
\hline Groupe V & $1(2)$ & & $2,7(6)$ & $3(1)$ & \\
\hline
\end{tabular}

Si nous examinons le Tableau 6 qui résume le taux de réussit et les intentions, nous pouvons observer la meme tendance. Ce tableau montre également qu'il y a des éleves (par exemple l'éleve $\mathrm{n}^{\circ} 20$ et $\mathrm{n}^{\circ} 23$ ) qui ont eu des difficultés avec le test, ils ne sont pas arrivés a un niveau élevé, mais ils aimeraient résoudre a nouveau des problemes de ce type. Il y a, bien sur, des éleves qui ont réussi a résoudre tous les niveaux de test, mais qui n'aimeraient pas poursuivre ces tests (les éleves $\left.n^{\circ} 7, n^{\circ} 10, n^{\circ} 52\right)$. Néanmoins leurs réponses aux questions suivantes montrent qu'ils sont ouverts a des problemes semblables mais de types différents. On peut également observer que parmi les 18 éleves qui ont choisi la réponse « Oui, s'il y a de nouveaux tests. »(3), 11 sont arrivé a la fin du test. Nous pouvons penser que pour eux ce test ne représente pas un nouveau défi c'est pourquoi ils aimeront retrouver autres types d'exercices.

Tableau 6. !!!!

\begin{tabular}{|l|l|l|r|r|}
\hline taux de réussit / intention & 1 & 2 & 3 & 4 \\
\hline Niveau 1 & 3 & 1 & & \\
\hline Niveau 2 & 2 & & 2 & \\
\hline Niveau 3 & 3 & 6 & 2 & 2 \\
\hline Niveau 4 & 1 & 1 & 3 & \\
\hline Niveau 5 & 3 & 1 & 11 & 2 \\
\hline
\end{tabular}

La deuxieme question portait sur la difficulté du test. Les réponses étaient numérotées de 1 a 5 , ou le 1 signifie «tres facile »et 5 signifie «tres difficile». Nous avons calculé les moyennes comme précédemment.

Dans le Tableau 7, nous pouvons observer que la tendance a considérer les exercices comme plus faciles croît avec l'âge. Ce fait est en accord avec les résultats obtenus : les éleves les plus âgés sont arrivés a un niveau plus élevé et ils ont eu moins de difficulté pendant la résolution des exercices. Le tableau nous montre 
Tableau \%. !!!!

\begin{tabular}{|l|r|r|r|r|r|}
\hline Groupe/niveau atteint & Niveau 1 & Niveau 2 & Niveau 3 & Niveau 4 & Niveau 5 \\
\hline Groupe I & & & $4(1)$ & $3,5(2)$ & $3,4(7)$ \\
\hline Groupe II & & $4(3)$ & $3(2)$ & $4(1)$ & $3,5(2)$ \\
\hline Groupe III & $4(2)$ & $5(1)$ & $2(2)$ & $1,5(2)$ & $2,5(2)$ \\
\hline Groupe IV & & & & & $2,8(6)$ \\
\hline Groupe V & $4,5(2)$ & & $3,3(6)$ & $2(1)$ & \\
\hline
\end{tabular}

aussi que, dans un groupe, les éleves qui ont le mieux réussi considerent que les exercices sont faciles.

A la fin du questionnaire nous avons énuméré cinq types de problemes :

- la composition de plusieurs réflexions,

- des exercices de rotation plus difficiles,

- une promenade sur le cube,

- des exercices semblables pour d'autres solides (tétraedre, octaedre),

- des exercices concernant les patrons du cube.

Nous avons demandé aux éleves quels étaient, parmi ces types de problemes, ceux qu'ils aimeraient résoudre. Les exercices dénommés « Promenade sur le cube »ont remporté les faveurs de plus grand nombre. Nous pensons que c'est plutôt la dénomination qui a été choisie car ils ne pouvaient pas savoir de quel type de probleme il s'agissait précisément.

Si nous analysons les réponses, nous pouvons observer que les éleves qui sont plus ouverts aux nouveaux types d'exercices ont eu de meilleurs résultats. Cependant des réponses proviennent de tous les niveaux. Selon ces réponses et les autres remarques comme « nagyon zsir »(tres bon, intéressant), « c'est une idée assez originale », nous pensons qu'il est intéressant de continuer a travailler dans ce sens.

\section{Conclusion}

Dans ce travail nous nous somme proposé d'examiner comment l'ordinateur peut intervenir dans le processus d'acquisition de la rotation mentale.

Un logiciel concernant les rotations d'un cube a été écrit. Nous avons essayé d'analyser si la capacité de «rotation mentale »des éleves peut etre améliorer a l'aide de ce logiciel. Les exercices ont été regroupés en cinq catégories. Le niveau de difficulté des exercices a été augmenté. Chaque probleme comporte trois 
dessins : le premier dessin est un cube ABCDEFGH dont les lettres sont mises par l'ordinateur dans des ordres différents; le deuxieme dessin est le dessin de la transformation en précisant le plan de réflexion ou l'axe et l'angle de rotation; le troisieme dessin est un cube ou a côté d'un sommet nous avons mis un point d'interrogation. En ce sommet les éleves devaient taper la lettre qui apres la transformation se trouvera dans cette position. Si l'éleve donne une réponse erronée l'ordinateur dessine la bonne réponse (le premier cube, la transformation et le cube avec la réponse).

La statistique montre que les résultats des éleves s'améliorent au fur et a mesure. En faisant plusieurs aller-retour entre le page original (qui contenait les réponses données par eux) et la page WEB avec les réponses correctes, les éleves ont réussi petit a petit de surmonter leurs erreurs.

$\mathrm{Si}$, avec l'utilisation des maquettes, l'éleve a appris les notions fondamentales, l'ordinateur pourrait alors l'aider a les approfondir. L'ordinateur est capable d'engendrer beaucoup d'exercices différents, au cours de leur résolution l'éleve pourrait se rendre compte de ses progres, d'autre part le professeur en dressant les bilans pourrait suivre les progres et les lacunes de ses éleves. Les résultats de l'expérience laissent penser que l'utilisation de l'ordinateur peut aider a approfondir un composant de la vision spatiale.

De nombreux types d'exercices sont facilement réalisables a l'aide de l'ordinateur. Les apprentissages visés ici pourraient se faire avec profit tout au long de la scolarité, et en particulier au college.

Pendant les expériences, trois éleves ont remarqué qu'ils avaient eu des difficultés avec l'interprétation de la position de la fleche montrant la direction de la rotation. Ils n'ont pas arrivé a discerner si la fleche passait au-dessus ou audessous de l'axe de rotation. Ces problemes peuvent etre résolus de plusieurs façons. Par exemple on pourrait utiliser pour la fabrication de tous les dessins, le logiciel PovRay qui dessine les figures en perspective avec trois centres, en portant l'attention sur le dessin des fleches. Le probleme avec cette méthode est que la dimension de la figure sera plus important et donc que le téléchargement sur les réseaux scolaire sera plus lent. L'autre possibilité serait l'utilisation des sens de rotations (droit ou gauche selon l'accord). Dans ce cas l'un coté de l'axe sera plus gros, l'éleve devrait prendre, en imagination, l'axe dans la main dans la façon que le pouce tombe sur ce côté de l'axe, la direction de la rotation sera indiquée par les autres doits (regle des trois doigts). 
Comme perspective de recherche nous avons pensé élaborer des logiciels avec lesquels nous pouvons aider a l'acquisition de la visualisation, les relations spatiales, et l'orientation.

\section{Références}

[1] G. Brousseau, Théorie des situations didactiques, Editions La Pensée sauvage, Grenoble, 1998.

[2] H. Chaachoua, Fonctions du dessin dans l'enseignement de la géométrie dans l'espace, Etude d'un cas : la vie des problèmes de construction et rapports des enseignants à ces problemes, These de doctorat, Grenoble, 1997.

[3] L. Cooper et R. Shepard, Le retournement mental des objets, Pour la Science $\mathbf{8 8}$ (1985), 40-47.

[4] N. Hara et R. Kling, Students' frustrations with WEB-Based Distance Education, First Monday 412 (1999), 40-47, http//firstmonday.org/issue4_12/hara/index.html.

[5] J. Hartmann et K. Reiss, Problem solving process in a spatial geometry environnement, Selected Papers from Annual Conference on Didactics of Mathematics, $(\mathrm{H}$. G. Weigand, A. Peter-Koop, N. Neill, K. Reiss, G. Törner et B.Wollring, eds.), Munich, 1998, 18-30.

[6] S. Kosslyn, Image and mind, Harvard University Press, 1980.

[7] P. H. Maier, Spatial geometry and spatial ability- How to make solid geometry solid? Selected Papers from the Annual Conference of Didactics of Mathematics 1996, (Elmar Cohors-Fresenborg, Hermann Maier, Kristina Reiss, Gounter Toerner, and Hans-Georg Weigand, eds.), Osnabrueck, 1998, 63-75.

[8] P. H. Maier, Raumgeometrie mit Raumvorstellung, Thesen zur Neustrukturierung des Geometrieunterrichts, 1999.

[9] B. Parzysz, K̈nowingüs S̈eeing, Problems of the plane representation of space geometry figures, Educational Studies in Mathematics 19 (1988), 79-92.

[10] B. Parzysz, Représentations planes et enseignement de la géométrie de l'espace au lycée, These de doctorat, Université Paris 7, 1989.

[11] K. Reiss, Spatial ability and declarative knowledge in a geometry problem solving context, Vol. I, Proceedings of 23rd International Conference for the Psyhology of Mathematics Education, (O. Zaslavsky, eds.), Technion, Haifa (Israel), 1999.

[12] R. Shepard, The mental image, In American Psychologist (1978), 125-137.

MÁRIA BAKÓ

LYCÉE FAZEKAS MIHÁLY DE DEBRECEN

HONGRIE

E-mail: aszalos@inf.unideb.hu

(Received .................) 